\title{
Operant reinforcement of a skeletally mediated autonomic response: Uncoupling of the two responses'
}

\section{ROCHELLE J. GAVALAS, BRAIN RESEARCH INSTITUTE, UNIVERSITY OF CALIFORNIA, LOS ANGELES, Los Angeles, Calif. 90024}

The mediation explanation of the effect of operant reinforcement on autonomic responses assumes that skeletally mediated autonomic responses can be easily and fortuitously conditioned. GSR deflections elicited by deep respirations were operantly reinforced. The two responses became uncoupled: the skeletal (deep respiration) response showed a classic instrumental learning curve, the autonomic response showed great variability and only marginal (E-C) gains during extinction. It is possible that these marginal gains were artifactual since the per cent of deep respirations eliciting GSRs showed a marked habituation to increasing repetitions of the respiration response.

Evidence has begun to accumulate showing that it is possible to directly, operantly reinforce autonomic responses (Gavalas, 1967; Miller, Trowill, DiCara, Carmona, \& Banuazizi, 1966; Crider, Shapiro, \& Tursky, 1967; Van Twyver \& Kimmel, 1966; and others). The most critical question in these studies has been that of controlling for the effect of possible covert mediating skeletal responses. Two techniques have been used to control for mediators an irrelevant task has been introduced into the experiment to control for subvocal and respiratory mediators (Gavalas, 1967) and curare has been used to eliminate skeletal activity (Miller \& DiCara, 1967; Trowill, 1967). In all of these studies, it was possible to demonstrate a modification in frequency of the autonomic response as a consequence of response contingent, operant reinforcement.

However, these data have been questioned. Black (1967) has asked whether or not the animals used by Miller et al were completely curarized. Kendler \& Kendler (1962) have argued that a purely theoretical or logical mediator may be used as an explanation provided that it is "useful" to do so.

The continued invocation of the mediation hypothesis as an ad hoc explanation of the effects of operant reinforcement on autonomic responses raises certain important issues. This kind of explanation is vague and in danger of being untestable in principle. No matter how many skeletal responses are eliminated or controlled, it can always be asserted that some other unobserved response is doing the mediating. The mediation hypothesis implies that operant reinforcement of a skeletally mediated autonomic response is highly effective. It assumes that such conditioning can occur easily and fortuitously within a great variety of experimental situations. Here we have attempted to explicate at least one simple form of the mediation hypothesis and make it empirically testable. In this way some of the burden of proof may be put back on the shoulders of the mediation theorists. In the present study GSR deflections which were known to be skeletally mediated were operantly reinforced. These data provide us with an opportunity to observe both the curve of a specified skeletal mediator and the curve of its mediated autonomic response.

It had been observed in an earlier study on operant reinforcement of nonspecific GSRs that deep yawn-like inspirations often elicited GSR deflections. This spontaneous, semi-voluntary response was well suited to the present study. GSR deflections which were elicited by these deep inspirations were-reinforced. (Fig. 1).

Method. The Ss were 10 female college-age paid volunteers. A Grass PGR apparatus which applies a constant current of $50 \mu \mathrm{A}$ to the skin was used with a frequency cutoff of $3 \mathrm{cps}$ to eliminate noise and artifacts. Standard Wenger electrodes (Sanborn) with zinc sulphate paste were used on the right palm of each S. Deflections as small as $500 \mathrm{ohms}$ could be reliably detected. Respiration was measured with a belt-type pneumatometer.

The $S$ sat quietly for $25 \mathrm{~min}$ while the electrodes were attached and the apparatus was calibrated; the learning session lasted 20 min each day. The $S$ was seated with arms comfortably extended on a small table in front of her and a lightweight sandbag was placed on her right arm to prevent movement artifacts. Directions were given via a loudspeaker, and the behavior of the $S$ was monitored with closed circuit TV. Ss were asked to sit quietly. They were told that this was a study of day-to-day changes in several physiological variables and each measuring device was explained to them. A tape recorded series of nonsense syllables was spelled aloud and they were asked to pronounce the syllables as they heard them (see Gavalas, 1967). The task acted as a control on subvocal mediators and as a control on level of attention. The reinforcer was a light-flash followed by the comment "that's good." Ss were told that their responses were being watched and the light would come on when they were doing well. No responses were reinforced (or counted) within $15 \mathrm{sec}$ of the reinforcer, to control for the inadvertent reinforcement of reinforcer-elicited responses. Elicited GSRs usually followed deep respirations with a characteristic latency; GSRs that greatly exceeded this latency were not classified as respiration-produced.

The first session served as an introduction to the experiment; no records were taken. On Day 2, the experiment was begun and operant levels were recorded. On Day 3 the reinforcer was presented randomly for $5 \mathrm{~min}$ and then made response-contingent for experimental Ss for the remaining $15 \mathrm{~min}$. On the succeeding three and one half sessions, deep inspirations immediately followed by GSR drops of $500 \mathrm{ohms}$ or more, were operantly reinforced. Extinction began on the last half of Day 7 and continued for the next three days. All Ss were run at the same hour each day.

Four control Ss were matched with the four experimental Ss on the basis of their Day 2 operant level rate of nonspecific GSR responding and yoked for schedules of reinforcement throughout the experiment to control for the stimulating effect of the reinforcer.

Generally, "deep respirations" were easy to count reliably (see Fig. 1). For those that were difficult to classify, the record for a given $S$ for a given day was scanned; four to five typical respirations were averaged and compared to one unmistakable deep respiration. Deep respirations were so classified if their amplitude exceeded the average respirations by $60 \%$ of the average-deep difference or more.

Results and Discussion. The average learning curves for the three responses are shown in Fig. 2. The skeletal response (deep respirations) of the experimentals follows the typical negatively accelerated learning curve usually seen for operant reinforcement of instrumental responses (Fig. 2A). There is a marked increase in frequency over time and a clear gain over the curve of the yoked controls. Nonspecific GSRs (Fig. 2C) show a decline for both experimentals and controls. Respiration elicited GSRs (Fig. 2B) follow a much more variable course than the other two responses. There appears to be a gain in frequency during extinction (Days 7 to 10), if experimentals are compared to controls. The mediated GSRs do not follow precisely the course of the mediator, however, and their absolute rate is considerably less; e.g., the Day 7 peak of mediated GSRs is only 0.35 per min while deep respirations on the same day reach 0.5 per min. The curve of the respiration elicited GSRs appears to be both a function of the increase in rate of the mediator and a function of the day-to-day decline of nonspecific responses.

The mediated autonomic response tends to become uncoupled from its skeletal mediator and this dissociation becomes more marked as the experiment continues. Fig. 3 shows per cent of deep

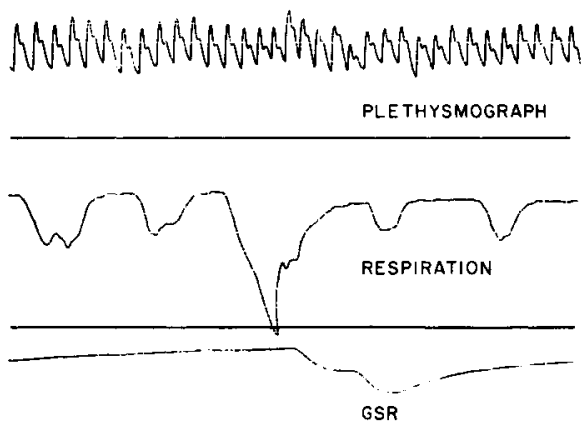

REINFORCEMENT

Fig. 1. Sample record of operant reinforcement of a skeletally mediated GSR deflection. 


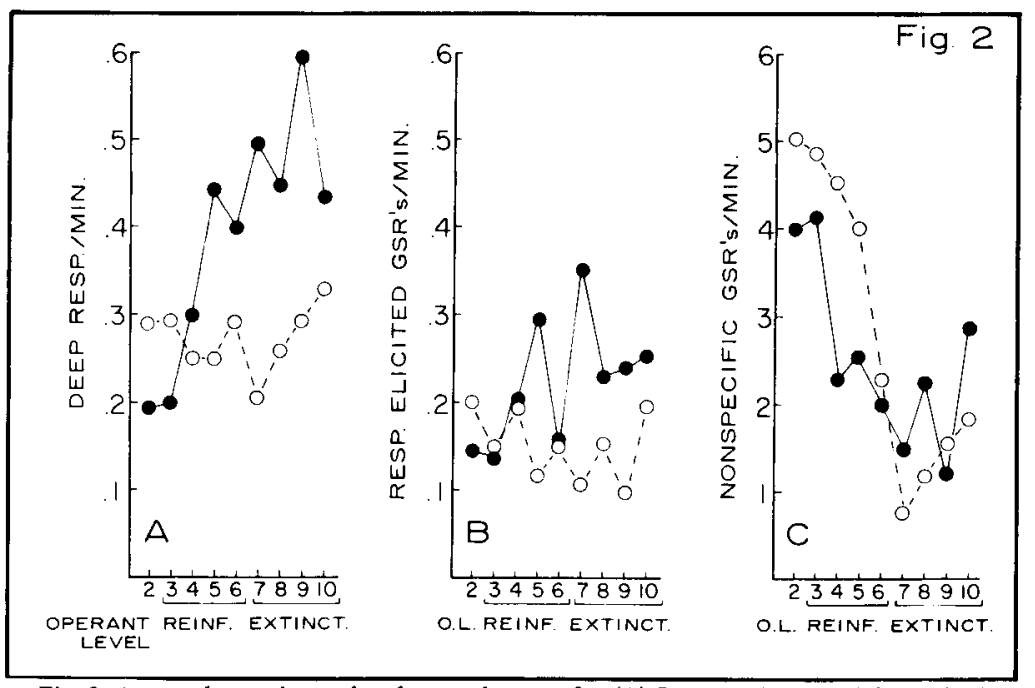

Fig. 2. Averaged experimental and control curves for (A) Deep respirations, (B) Respiration elicited GSRs, and (C) Nonspecific GSRs. Solid lines are experimental curves; dashed lines are controls.

respirations that elicit GSR deflections plotted as a function of number of deep respirations emitted; it may be seen that both experimentals and controls show a marked habituation. It is possible that if this curve were extended, elicited GSRs would drop out altogether. This did occur in the individual curve of one experimental S. It is a question for further experimentation. The adaptation of the elicited GSR to repetitions of the skeletal response resembles the habituation of GSR deflections to external stimuli (such as loud noises) observed by Davis (1934) and others. These results challenge the assumption that skeletally mediated autonomic responses can be easily and fortuitously conditioned by operant reinforcement. Indeed, the orderly habituation of the mediated response to the mediator raises the possibility that the experimental-control differences of Fig. 2B are artifactual and do not represent learning at all. It may be that with extended conditioning trials the elicited GSRs would have completely habituated to the occurrence of the skeletal mediator.

The generality of these results to other autonomic responses and other mediators remains to be established. Shapiro \& Shneidman (1968) have observed, similarly, that operant reinforcement of GSRs elicited by external stimuli is difficult to establish. It is possible that the present findings reflect the fact that the skeletal mediator in this experiment was relatively "intense" and easy to discriminate while the autonomic response in question was relatively small and difficult to discriminate.

GSR as an orienting reflex. If the GSR in this experimental situation is viewed as an orienting reflex, these results may be interpreted as showing that operant reinforcement could not prevent the inhibition of the mediated GSR during the elaboration of the skeletal response.

If the deep respirations and the GSR are viewed as two aspects of one global orienting reflex, then the present results may be interpreted as showing that operant reinforcement of an orienting reflex results in the uncoupling of component responses of the reflex.

Implications for two-factor theories of learning. These results support Skinner's (1938) view that the susceptibility of a response to operant reinforcement depends upon whether or not it is correlated with an observable, external stimulus. GSR deflections that were not correlated with an observable stimulus (Gavalas, 1967) could be modified by operant reinforcement. In the present study, GSR deflections that were correlated with an observable stimulus (the stimulus components of the respiratory, skeletal response) could not be easily modified by operant techniques. This is precisely what Skinner predicts. This, in turn, suggests that the autonomicskeletal classification of responses (Mowrer, 1960; Konorski \& Miller, 1937) is not the most satisfactory basis for predicting the mode of conditioning to which a given response is susceptible.

These findings suggest some hypotheses about the course of response-elicited autonomic responses during simple operant rein-

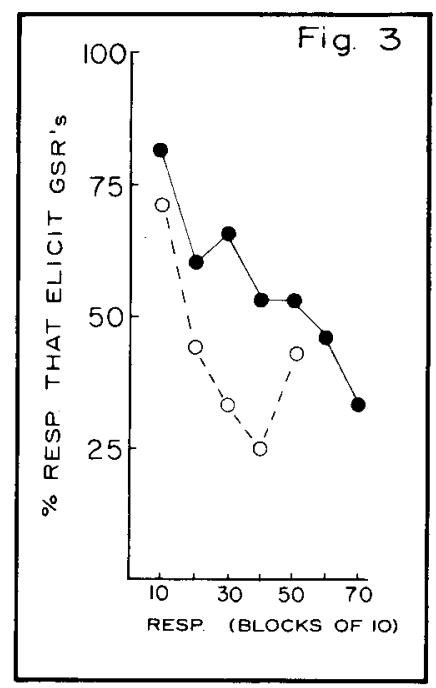

Fig. 3. Habituation of elicited GSRs to repetitions of the respiration response.

forcement of a skeletal response. It seems likely that the autonomic components ("hope"; "fear") of the skeletal response could not be easily and "accidentally" established within a simple instrumental learning situation as Mowrer (1960) has suggested.

Direct operant reinforcement of autonomic responses has been questioned on the basis of whether or not covert skeletal mediators could account for the results. In this experiment, we have attempted to test one simple version of the mediation hypothesis-selective operant reinforcement of an autonomic response as it is elicited by a single, observable skeletal response. The results show that under these conditions the skeletal response shows a learning effect but the autonomic component tends to become dissociated from its mediator. The autonomic response shows an orderly habituation to increasing repetitions of the skeletal response.

\section{REFERENCES}

BLACK, A. H. Transfer following operant conditioning in the curarized dog Science, 1967, 155, 201-203.

CRIDER, A., SHAPIRO, D., \& TURSKY, B. Reinforcement of spontaneous electrodermal activity.J. comp. physiol. Psychol., 1966, 61, 20-27.

DAVIS, R. C. Modification of the galvanic reflex by daily repetition of a stimulus. J. exp. Psychol., 1934, 17, 504-535.

GAVALAS, R. J. Operant reinforcement of an autonomic response: two studies. J. exp. Anal. Behav., 1967, 10, 119-130.

KENDLER, H. H., \& KENDLER, T. S. Vertical and horizontal processes in problem solving. Psychol. Rev., 1962, 69, 1-16.

KONORSKI, J., \& MILLER, S. On two types of conditioned reflex. J. genet. Psychol, 1937, 16, 264-272.

MILLER, N. E., \& DiCARA, L. Instrumental learning of heart rate changes in curarized rats: shaping and specificity to discriminative stimulus. $J$. comp. physiol. Psychol., 1967, 63, 12-19.

MILLER, N. E., TROWILL, J., DiCARA, L., CARMONA, A., \& BANUAZIZI A. Extending the domain of learning. Science, 1966, 152,676 (Abstract).

MOWRER, O. H. Learning theory and behavior. New York: Wiley, 1960.

SHAPIRO, D., \& SHNEIDMAN, B. Personal communications, 1968.

SKINNER, B. F. The behavior of organisms: an experimental analysis. New York: Appleton-Century, 1938.

TROWILL, J. A. Instrumental conditioning of the heart rate in the curarized rat.J. comp. physiol. Psychol., 1967,36,7-11.

VAN TWYVER, H. B., \& KIMMEL, H. D. Operant conditioning of the GSR with concomitant measurement of two somatic variables. J. exp. Psychol, $1966,72,841-846$

NOTE

1. This study was supported by the Air Force Office of Scientific Research of the Office of Aerospace Research, under Contract No. AF 49(638)-1387, and Contract NONR 233(91) between the Office of Naval Research, Department of the Navy and the Brain Research Institute of the University of California, Los Angeles, and the National Aeronautics and Space Administration under Grant No. NsG 237-62.

Appreciation is expressed to R. T. Kado for advice and instrumentation of the project. I am indebted to Barbara Vosen for her technical assistance and for giving unstint ingly of her time. 